\title{
Awareness of Breast Cancer Screening among the Medical and General Population of the North Region of Cameroon
}

\author{
Richard Tagne Simo $\mathbb{D D}^{1}{ }^{1}$ Erika Myriam Baiguerel, ${ }^{1}$ Armel Hervé Nwabo Kamdje ${ }^{1 D},{ }^{1}$ \\ Paul Faustin Seke Etet ${ }^{\circ},{ }^{2}$ Mohamadou Ahmadou, ${ }^{1}$ Charlette Nangue, ${ }^{3}$ \\ and Phelix Bruno Telefo ${ }^{4}$ \\ ${ }^{1}$ Department of Biomedical Sciences, Faculty of Science, University of Ngaoundere, Cameroon \\ ${ }^{2}$ Department of Physiological Sciences and Biochemistry, FMBS, Garoua, University of Ngaoundere, Cameroon \\ ${ }^{3}$ Anatomo-Cytopathology Laboratory, University Hospital Center of Yaounde, Cameroon \\ ${ }^{4}$ Department of Biochemistry University of Dschang, Cameroon
}

Correspondence should be addressed to Richard Tagne Simo; richardsimotagne@yahoo.fr

Received 21 November 2020; Accepted 8 July 2021; Published 27 July 2021

Academic Editor: Pranshu Sahgal

Copyright (c) 2021 Richard Tagne Simo et al. This is an open access article distributed under the Creative Commons Attribution License, which permits unrestricted use, distribution, and reproduction in any medium, provided the original work is properly cited.

\begin{abstract}
Breast cancer has become a real public health problem in Cameroon, particularly in rural areas due to late diagnosis, resulting partly from the absence of national screening programs. This work is aimed at assessing breast cancer awareness in the North Region of Cameroon. Participants were selected in six health centers surrounding the rural area of Garoua, North Region, Cameroon, and administered a questionnaire aimed at assessing their awareness about breast cancer risk factors and screening. Out of the 475 women (including 37 medical personnel) interviewed, 45.5\% attended at least secondary school; 91.3\% were aware of the disease with the main sources of information from those around them (64.8\%), media (46.5\%), and health professionals in health facilities (42.7\%). 23.3\% had misconceptions and myth-based ideas on the origin of the disease. Ignorance was the main reason preventing the performance of breast self-examination, and the high cost prevents individuals from going for mammography. The highest awareness rate was observed in employed women with higher level of education. Our study highlights the need to raise awareness among the populations in North Region, Cameroon, about the risk factors and clinical signs of breast cancer and the importance of screening practice for early diagnosis of breast cancer.
\end{abstract}

\section{Introduction}

Breast cancer is a major public health challenge worldwide, as it is the leading cause of cancer-related deaths among women, particularly in developing countries $[1,2]$. About 2.1 million women were diagnosed with breast cancer in 2018, and 626,679 breasts cancer-related deaths were reported. These represented an increase rate of $19 \%$ in the incidence and $17 \%$ in the mortality rates as compared to 2012 [1, 3-5]. In Africa, breast cancer represented 16\% of cancer incidence and $18.3 \%$ of cancer-related mortality in 2018. In Cameroon, the situation is becoming alarming as breast cancer-related mortality rate displayed an increase of 34\% between 2012 and 2018. 3,273 new cases were diagnosed and 1,780 women died of breast cancer in 2018 , ranking it the cancer with the highest incidence and highest death toll in Cameroon $[1,6]$. Also alarming is the fact that the number of women younger than 35 diagnosed with severe highgrade breast cancer has been increasing [7-9]. The five-year median survival rate is $22 \%$, and most patients die within 12 months after diagnosis [10-13].

Breast cancer generally has a favorable prognosis in developed countries, partly due to detection at early and asymptomatic stages, thanks to the establishment of screening practices and therapeutic advances [14, 15]. Tools like the widely recognized Breast Cancer Risk Assessment Tool (BCRAT or Gail model) that were developed and adapted for the disparity in racial and ethnic groups in developed 
countries, in particular in the United States, have been proving to be challenging to adapt to developing countries $[16,17]$, including Cameroon, where information about the epidemiology and diversity of dominant risk factors of breast cancer in female populations is often scarce $[8,18]$.

In Cameroonian cities, few women attend national screening programs when available, partly due to the lack of awareness about breast cancer and screening methods $[19,20]$. In addition, unfortunately, mammography is still inaccessible to the majority of the population due to high costs. Thus, cases with breast lumps are detected most often accidentally and more than $80 \%$ of cases are diagnosed in late stages (stages III and IV) where the disease usually has a poor prognosis [7,9]. This explains why, in most African countries like Cameroon, breast cancer survival rate is low. Thus, breast cancer screening among population should be a priority in this country, particularly in rural areas where healthcare centers are lacking [21-23], considering that breast cancer incidence is expected to be different as compared to cities due to environmental differences [24-26].

The present study was aimed at assessing breast cancer awareness in the North Region of Cameroon, to contribute to the identification of the factors underlying the increased incidence and late diagnosis of breast cancer observed recently in Cameroon.

\section{Materials and Methods}

2.1. Participants and Ethical Considerations. Our study participants were 475 women randomly selected among the residents of the rural areas around the city of Garoua, North Region, Cameroon. Signed informed consent was obtained from each participant at recruitment. Before analysis, data collected were anonymized to protect the privacy of participants. All the procedures of the study were approved by the North Regional Delegation of the Ministry of Health.

2.2. Study Procedures. From August to December 2019, a cross-sectional and descriptive study was conducted in six health facilities of the North Region of Cameroon. Participants were recruited in the health facilities using simple random sampling. Women previously diagnosed with breast cancer and women undergoing unilateral or bilateral mastectomy were not included.

After being presented individually with the study rationale and detailed information on procedures, each participant willing to join the study had to sign an informed consent form. Afterwards, she was administered a questionnaire which was aimed at assessing awareness about breast cancer and screening practice and its risk factors and collecting some sociodemographic data. Then, physical breast examination was performed.

2.3. Data Collection. Awareness about breast cancer, associated risk factors, and recommended screening methods was assessed using a questionnaire collecting the following information: (i) Demographic characteristics like age, education, and occupation

(ii) Physiological factors with established risk potential in breast cancer context such as age (to assess whether age was advanced), age at first menarche (to determine early onset), and the presence and onset of menopause (late vs. early)

(iii) Personal history and family-related factors such as parity (to distinguish nulliparous from multiparous), age at first pregnancy (to determine whether it occurred late), history of breastfeeding, history of exposure to ionizing radiation, history of hormonal treatment, history of OCP use, family history of breast cancer, and history of alcohol and tobacco consumption

In addition, the questionnaire also included questions aimed at assessing

(i) the awareness about the recommended screening methods for breast cancer early detection (breast self-examination and mammography)

(ii) knowledge about commonly reported breast cancer symptoms like nipple discharge, change in skin appearance, change in breast size, the presence of breast masses, and inflamed axillary lymph nodes

2.4. Breast Physical Examination. When a nodule was identified as well as its characteristics (site, number, consistency, size, mobility relative to deep skin and plans, and painful or not) during physical breast examination performed in the gynecology service of the health facility, fine-needle aspiration (FNA) was performed with the consent of the participant, using recommended standard clinical procedures. Each sample collected was mounted on a slide, processed for May Grünwald-Giemsa staining and analyzed using bright-field microscopy (magnifications $4 \mathrm{x}$ to $40 \mathrm{x}$ ) at the Laboratory of Anatomo-Cytopathology of the University Hospital Center of Yaoundé. In case of cancer, Fisher's simplification of Black's nuclear grading scheme was used to determine the grade.

Alternatively, when nipple discharge occurred during the examination, characteristics were determined and recorded as well (aspect, uni- or bilateral, and uni- or multiorifice). Moreover, palpation of the armpits was also performed to assess eventual axillary lymph node inflammation. The following characteristics of inflamed axillary lymph nodes were determined and recorded: number, size, and mobility relative to surrounding tissue.

2.5. Statistical Analysis. Data analysis was performed using XLStat Version 2019. The $\chi^{2}$ test was used to compare proportions, and correlations were determined between education, occupation, and breast cancer awareness and also between education, occupation, and breast cancer screening awareness and its practice. Statistical significance was set at $p<0.05$. 
TABLE 1: Sociodemographic characteristics of participants.

\begin{tabular}{lccc}
\hline Variables & & $N$ & $\%$ \\
\hline \multirow{2}{*}{ Age (years) } & $<35$ & 403 & 84.6 \\
& $\geq 35$ & 72 & 15.1 \\
\hline \multirow{3}{*}{ Education } & No formal & 95 & 20.0 \\
& Primary school & 111 & 23.3 \\
& Secondary school & 221 & 46.5 \\
& University & 48 & 10.1 \\
\hline \multirow{3}{*}{ Occupation } & Housewife & 175 & 36.8 \\
& Student & 118 & 24.8 \\
& Officer & 24 & 5.0 \\
& Health professional & 37 & 7.7 \\
& Other, informal sector & 121 & 25.4 \\
\hline
\end{tabular}

\section{Results}

3.1. Sociodemographic Information. 475 women were involved in this study, of which 37 (7.7\%) were health professionals. Table 1 shows the sociodemographic characteristics of participants. The age range was 13 to 72 years old, and the median was 42.5 . Most participants were younger than 35 (84.6\%), had attended at least secondary school (56.6\%), and were not housewives (63.2\%) (Table 1).

3.2. Awareness about Breast Cancer Risk Factors and Early Signs. Table 2 presents the distribution of participants according to their knowledge about breast cancer: $91.3 \%$ had heard about breast cancer and their main sources of information were their entourage $(64.8 \%)$, media $(46.5 \%)$, and health professionals (42.7\%) (Table 2). More than $50 \%$ of the population was not aware of breast cancer risk factors. Most of them had misconceptions and myth-based ideas such as traditional breast massage $(10.1 \%)$ and extended wearing of bra $(13.2 \%)$ as the origin of the disease (Table 2). Hereditary factors (12.8\%) and consumption of alcohol and tobacco (12.4\%) were the most known actual risk factors of breast cancer (Table 2). Breast lumps (43.7\%), pain (36.2\%), and wounds $(24.4 \%)$ were the most known breast cancer symptoms (Table 2 ).

3.3. Awareness about Breast Cancer Screening. Table 3 shows the distribution of participants according to their knowledge of breast cancer screening and the frequency of practice. $56.8 \%$ were aware of breast cancer screening and named clinical examination of the breast (34.9\%), BSE (27.7\%), and mammography (21.2\%) as screening tools (Table 3). However, only $4.6 \%$ already performed mammography and $24.0 \%$ practiced BSE, of which $31.5 \%$ (7.5\% of the overall participants) do so regularly (Table 3 ).

\subsection{Factors Affecting Breast Cancer Awareness and Screening} Practice Frequency. Participants had to choose among 10 choices on risk factors of breast cancer (including two erroneous concepts) and 5 choices on clinical signs/symptoms and the screening tools. Table 4 shows the association between occupation and educational level of participants and their knowledge regarding breast cancer. Highly edu-
TABLE 2: Breast cancer awareness and known risk factors and symptoms reported.

\begin{tabular}{|c|c|c|c|}
\hline Variables & & $N$ & $\%$ \\
\hline Awareness & Heard about breast cancer & 434 & 91.3 \\
\hline \multirow{4}{*}{$\begin{array}{l}\text { Information } \\
\text { source }\end{array}$} & Media & 221 & 46.5 \\
\hline & Friends & 308 & 64.8 \\
\hline & Health professionals & 203 & 42.7 \\
\hline & School & 71 & 15.7 \\
\hline \multirow{11}{*}{ Risk factors } & None & 233 & 49.0 \\
\hline & Hereditary & 61 & 12.8 \\
\hline & Prolonged use of pills & 29 & 6.1 \\
\hline & Alcohol and tobacco & 59 & 12.4 \\
\hline & Age & 13 & 2.7 \\
\hline & Obesity & 9 & 1.8 \\
\hline & Prolonged exposure to the sun & 16 & 3.3 \\
\hline & Low breastfeeding & 23 & 4.8 \\
\hline & Low deliveries & 7 & 1.4 \\
\hline & Traditional breast massage & 48 & 10.1 \\
\hline & Extended wearing of tight bra & 63 & 13.2 \\
\hline \multirow{6}{*}{ Symptoms } & None & 170 & 35.7 \\
\hline & Lump in the breast & 208 & 43.7 \\
\hline & Pain in the breast & 172 & 36.2 \\
\hline & Wound on the breast & 116 & 24.4 \\
\hline & Changes in breast shape and appearance & 108 & 22.7 \\
\hline & Nipple discharge & 61 & 12.8 \\
\hline
\end{tabular}

TABLE 3: Breast cancer screening methods cited and personal screening frequency.

\begin{tabular}{lccc}
\hline Variables & & $N$ & $\%$ \\
\hline Awareness & Heard about screening & 270 & 56.8 \\
\hline \multirow{2}{*}{ Screening methods } & None & 32 & 6.7 \\
& Clinical examination $^{\mathrm{a}}$ & 166 & 34.9 \\
& BSE $^{\mathrm{a}}$ & 132 & 27.7 \\
& Mammography & 101 & 21.2 \\
Screening practice & Ultrasound $^{*}$ & 89 & 18.7 \\
\hline \multirow{3}{*}{ BSE practice frequency } & Mammography & 22 & 4.6 \\
& BSE & 115 & 24.2 \\
\hline
\end{tabular}

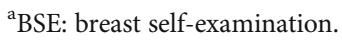

cated women were more likely to identify breast cancer actual risk factors (Table 4). However, they also had misconceptions and myth-based ideas: of the 48 women who attended university, $14(29.1 \%)$ and $13(27.0 \%)$, respectively, mentioned traditional breast massage and extended wearing of tight bra as risk factors and $15(31.2 \%)$ had no idea (Table 4$)$. Similarly, employed women were more aware of the disease and its risk factors and symptoms as compared to other groups 
TABLE 4: Associations between occupation, education, and breast cancer awareness.

\begin{tabular}{|c|c|c|c|c|c|c|c|c|c|c|c|c|c|}
\hline \multirow[t]{2}{*}{ Variables } & \multicolumn{5}{|c|}{ Occupation $(n)$} & \multirow[t]{2}{*}{ Total } & \multirow[t]{2}{*}{$p$} & \multicolumn{4}{|c|}{ Education $(n)$} & \multirow[t]{2}{*}{ Total } & \multirow[t]{2}{*}{$p$} \\
\hline & $\mathrm{O} 1$ & $\mathrm{O} 2$ & $\mathrm{O} 3$ & $\mathrm{O} 4$ & O5 & & & E1 & E2 & E3 & $\mathrm{E} 4$ & & \\
\hline \multicolumn{14}{|c|}{ Risk factors $\left(\mathrm{df}=437, \chi^{2}=91\right)$} \\
\hline None & 110 & 52 & 7 & 4 & 60 & 233 & $0.0001^{* * *}$ & 61 & 61 & 96 & 15 & 233 & $0.0002^{* * *}$ \\
\hline Hereditary & 5 & 20 & 7 & 21 & 8 & 61 & $0.0001^{* * *}$ & 1 & 4 & 40 & 16 & 61 & $0.0001^{* * *}$ \\
\hline$\uparrow$ Use of pills & 2 & 9 & 4 & 10 & 4 & 29 & $0.0001^{* * *}$ & 0 & 2 & 18 & 9 & 29 & $0.0001^{* * *}$ \\
\hline Alcohol/tobacco & 7 & 28 & 1 & 16 & 7 & 59 & $0.0001^{* * *}$ & 0 & 4 & 43 & 12 & 59 & $0.0001^{* * *}$ \\
\hline Age & 2 & 2 & 2 & 5 & 2 & 13 & $0.0002^{* * *}$ & 0 & 2 & 8 & 3 & 13 & 0.116 \\
\hline Obesity & 2 & 1 & 1 & 5 & 0 & 9 & $0.0001^{* * *}$ & 0 & 2 & 4 & 3 & 9 & 0.080 \\
\hline$\uparrow$ Sun exposure & 3 & 5 & 1 & 4 & 3 & 16 & 0.079 & 0 & 1 & 12 & 3 & 16 & $0.023^{*}$ \\
\hline$\downarrow$ Breastfeeding & 4 & 6 & 1 & 10 & 2 & 23 & $0.0001^{* * *}$ & 0 & 1 & 17 & 5 & 23 & $0.001^{* *}$ \\
\hline$\downarrow$ Parity & 2 & 2 & 0 & 2 & 1 & 7 & 0.306 & 0 & 1 & 5 & 1 & 7 & 0.433 \\
\hline Breast massage & 4 & 23 & 4 & 13 & 4 & 48 & $0.0001^{* * *}$ & 0 & 3 & 31 & 14 & 48 & $0.0001^{* * *}$ \\
\hline Tight bra wearing & 4 & 29 & 8 & 13 & 9 & 63 & $0.0001^{* * *}$ & 0 & 4 & 46 & 13 & 63 & $0.0001^{* * *}$ \\
\hline \multicolumn{14}{|c|}{ Symptoms $\left(\mathrm{df}=437, \chi^{2}=91\right)$} \\
\hline None & 87 & 19 & 1 & 1 & 62 & 170 & $0.0001^{* * *}$ & 53 & 65 & 52 & 0 & 170 & $0.0001^{* * *}$ \\
\hline Breast lump & 40 & 77 & 22 & 34 & 35 & 208 & $0.0001^{* * *}$ & 9 & 22 & 134 & 43 & 208 & $0.0001^{* * *}$ \\
\hline Breast pain & 52 & 60 & 14 & 23 & 23 & 172 & $0.0001^{* * *}$ & 15 & 29 & 100 & 28 & 172 & $0.0001^{* * *}$ \\
\hline Breast wound & 42 & 22 & 7 & 13 & 32 & 116 & 0.289 & 20 & 28 & 53 & 15 & 116 & 0.602 \\
\hline Br. appearance & 27 & 32 & 10 & 16 & 23 & 108 & $0.0002^{* * *}$ & 7 & 16 & 68 & 17 & 108 & $0.0001^{* * *}$ \\
\hline Nipple discharge & 9 & 25 & 6 & 9 & 12 & 61 & $0.0001^{* * *}$ & 3 & 7 & 42 & 9 & 61 & $0.0001^{* * *}$ \\
\hline Screening awareness & 77 & 81 & 22 & 37 & 33 & 270 & $\begin{aligned} \mathrm{df} & =12 ; \chi^{2}=74 \\
p & <0.0001^{* * *}\end{aligned}$ & 25 & 47 & 154 & 44 & 270 & $\begin{array}{c}\mathrm{df}=9 ; \chi^{2}=91 \\
p<0.0001^{* * *}\end{array}$ \\
\hline \multicolumn{14}{|c|}{ Screening methods $\left(\mathrm{df}=8 ; \chi^{2}=51.0\right)\left(\mathrm{df}=6 ; \chi^{2}=97.7\right)$} \\
\hline $\mathrm{BSE}^{\mathrm{a}}$ & 24 & 38 & 17 & 33 & 20 & 132 & $0.0001^{* * *}$ & 6 & 15 & 74 & 37 & 132 & $0.0001^{* * *}$ \\
\hline $\mathrm{CBE}^{\mathrm{b}}$ & 65 & 31 & 8 & 22 & 40 & 166 & $0.028^{*}$ & 22 & 39 & 83 & 22 & 166 & $0.056^{*}$ \\
\hline Mammography & 16 & 33 & 11 & 25 & 16 & 101 & $0.0001^{* * *}$ & 4 & 13 & 56 & 28 & 101 & $0.0001^{* * *}$ \\
\hline Ultrasound & 17 & 33 & 11 & 16 & 12 & 89 & $0.0001^{* * *}$ & 4 & 11 & 54 & 20 & 89 & $0.0001^{* * *}$ \\
\hline
\end{tabular}

${ }^{\mathrm{a} B r e a s t}$ self-examination; ${ }^{\mathrm{b}}$ clinical breast examination. $\mathrm{O} 1$ = housewife; $\mathrm{O} 2$ = student; $\mathrm{O} 3=$ officer; $\mathrm{O} 4=$ health professional; $\mathrm{O} 5=$ other informal sector; E1 = no formal education; E2 = primary school; E3 = secondary school; E4 = university; $\chi^{2}:{ }^{*} p<0.05 ;{ }^{* *} p<0.005 ;{ }^{* * *} p \leq 0.0001$.

$(p<0.0001)$ for most of the risk factors and symptoms (Table 4). Health professionals displayed better knowledge about breast cancer, although a few also had erroneous ideas and misconceptions (Table 4). Participants with higher education and employed women were also more aware of breast cancer screening, including breast self-examination (BSE), mammography, and ultrasound ( $p<0.0001$ vs. other groups) (Table 4).

In addition, the factors affecting breast self-examination (BSE) practice among the study participants are presented in Table 5. Breast cancer screening and BSE were mostly performed by women with higher education and employed women ( $p<0.0001$ vs. other groups) (Table 5).

3.5. Breast Examination Findings. Table 6 shows the results of the breast examination. Out of 475 participants, 329 agreed to undergo breast clinical examination as part of breast cancer screening. The following cases were detected: 23 with lumps, 3 with inflamed axillary lymph nodes, 2 with wounds on the breast, and 2 with wounds on the nipple
(Table 6). Analyses of fine-needle aspirates of nodules and lesions revealed 5 cases with the following cytological findings: a benign breast tumor, a galactophoric cyst, a cystic abscess, an abscess markedly swollen, and low chronic inflammation.

3.6. Reasons for Not Practicing Screening. Participants mainly claimed (i) a lack of awareness about breast cancer screening methods and fear of discovering that they are sick, (ii) lack of time for screening, (iii) that should it be breast cancer it could only result from witchcraft and thus they would not need hospitals to deal with it, and (iv) that they do not have any complaint and no history of breast cancer in their family, so there is no need for any exam. Other astonishing reasons proposed to reject screening included the following: (i) "Breast cancer results from keeping coins in bra and I never do so"; (ii) "This lump in my breast has always been there and never had a problem or any complaint with it, so there is no need for a test"; (iii) "I do not want to know whether my lump is or is not cancer, because it could result in stress, 
TABLE 5: Factors affecting BSE practice.

\begin{tabular}{lccccc}
\hline Variables & Never & $\begin{array}{c}\text { BSE practice }(n) \\
\text { Sometimes }\end{array}$ & Often & Total $(n)$ & $p$ value/df/ $\chi^{2}$ \\
\hline Educational level & 77 & 9 & 4 & 95 & $<0.0001^{* * *} / 9 / 47.1$ \\
None & 84 & 8 & 6 & 111 & \\
Primary & 147 & 44 & 17 & 221 & \\
Secondary & 20 & 18 & 9 & 48 & $<0.0001^{* * *} / 9 / 47.1$ \\
University & & & & 157 & \\
Occupation & 130 & 18 & 9 & 37 & \\
Housewife & 7 & 21 & 9 & 24 & \\
Health professional & 14 & 7 & 3 & 116 & \\
Officer & 86 & 20 & 10 & 114 & \\
Students & 91 & 13 & & & \\
Other informal sectors & 81 & &
\end{tabular}

$\chi^{2}:{ }^{* * *} p<0.0001 ; \mathrm{df}=$ degree of freedom.

TABLE 6: Clinical examination findings.

\begin{tabular}{lcc}
\hline Clinical aspect of the breast & $N$ & $\%$ \\
\hline Normal breasts & 296 & 89.9 \\
Nodules & 23 & 5.2 \\
Axillary lymph nodes & 3 & 0.9 \\
Umbilical nipple & 2 & 0.6 \\
Nipple discharge & 1 & 0.3 \\
Wound on the breast & 2 & 0.6 \\
Wound on the nipple & 2 & 0.6 \\
\hline
\end{tabular}

and I will be rejected by my husband if my breast is removed"; (iv) "No one in my family ever had breast cancer and I never had any complaint, that is why I will not perform any screening"; and $(v)$ "I do not know how to perform BSE, nobody ever taught me."

\section{Discussion}

Despite decades of laboratory, epidemiological, and clinical research, breast cancer incidence continues to rise $[27,28]$. This study aimed at assessing breast cancer awareness in the North Region reveals the existence of poor awareness about breast cancer's risk factors and its screening methods among women in the North Region of Cameroon. More than $50 \%$ of the population had no knowledge about breast cancer risk factors. Most of them had misconceptions about traditional breast massage (10.1\%) and extended wearing of bra $(13.2 \%)$ as risk factors. These results are surprising, given that most of the study participants have heard about breast cancer (91.6\%), out of which some were young and had the right education level to access more information $(46.5 \%$ attended at least secondary school). This could be explained by the fact that many of them heard about the disease from those around them such as friends and family members (64.8\%). Indeed, in traditional African public beliefs, breast cancer is a mystical disease resulting from supernatural causes, including witchcraft, curses, and divine punishment $[12,23,29]$.
The clinical signs most known by women were the presence of a lump in the breast (43.7\%), breast pain (34.0\%), and skin ulceration on the breast (23.5\%), as reported in previous studies [7, 30]. Indeed, due to poverty, in the North Region of Cameroon, patients generally consult when breast ulceration is observed or breast lumps are painful, and these signs usually occur when the disease is already at late stages [10-13], contributing to late detection of the disease and poor prognosis $[7,9]$.

In the present study, highly educated and employed women identified breast cancer risk factors more accurately than uneducated and unemployed women $(p<0.0001)$. However, misconceptions on the origin of the disease were also found here. Of the 48 women with university degrees, $15(31.2 \%)$ had no idea about disease risk factors, and 14 (29.1\%) and $13(27.0 \%)$, respectively, mentioned traditional breast massage and extended wearing of tight bra as risk factors. These women revealed that their erroneous information came from their entourage, and they did not see the importance of verifying it before participating in the study. This further suggests a lack of public awareness about breast cancer and underlines the need for education about early diagnosis and better prognosis of the disease. Media could help in disseminating the right information, as they are a source of information for $46.5 \%$ of the participants. Health facilities could help as well, as $28.7 \%$ of the participants reported that the health education sessions in local languages held during prenatal consultations and immunization days in Cameroonian healthcare centers are their major source of medical information. Studies in comparable contexts showed that presentations in local languages by qualified health professionals attract a large audience $[29,31]$. As expected, in this study, most health professionals interviewed (and all those working with breast cancer patients) were aware of breast cancer risk factors and listed the clinical signs of the disease accurately. However, only $24.32 \%$ practiced BSE regularly, $10.81 \%$ had already undergone mammography screening as a medical prescription, and none had ever undergone mammography as routine screening. This observation corroborates reports in other African countries [31,32] and suggests that 
good knowledge does not imply better screening practices. This was explained by negligence or the high costs of mammography.

Similarly, several other participants reported that it is possible to detect breast cancer early and cited clinical breast examination (32.87\%), breast self-examination (22.60\%), and mammography as screening tools. However, a low level of practice of mammography screening (4.11\%) and BSE (6.16\%) was also observed here, also due to high cost of mammography, together with a lack of mastery of the breast self-examination (BSE) technique and the fear of actually discovering a disease sign, considered a harbinger of mastectomy and death $[12,19,29]$. Considering the alarming rates of disease also reported elsewhere in the country, the Cameroonian Government should make mammography, the current gold standard for breast cancer screening, available for free to rural populations, for instance, through screening campaigns. BSE, a low-cost screening option which proved to be effective in similar contexts $[1,33]$, could be examined and recommended to women during these campaigns as it is the case in various successful breast cancer screening programs worldwide $[14,17,22,34]$.

Surprisingly, 79 (16.63\%) participants refused clinical examination in this study, as they feared discovering breast cancer or to show their breasts to the medical professionals performing the examinations due to religious reasons (to preserve their intimacy). This also justified the refusal of several participants to be sampled for cytopathological examination of the nodules, as they argued that they needed the consent of their husbands. Breast palpation coupled with fine-needle aspiration, a cost-effective cytopathological analytic technique efficient in this context [35], revealed nodules in $4.55 \%$ of the participants, confirming the need for breast cancer screening campaigns in Cameroonian rural areas like the North Region.

\section{Conclusion}

Our study highlights the need to raise awareness among the public about the risk factors and clinical signs of breast cancer and the importance of screening practice by both mammography and regular practice of BSE and CBE in the diagnosis of breast cancer at early stage and management of the disease. The Cameroonian health authorities should organize mammography screening campaigns for an early detection of asymptomatic cases of breast cancer, considering the positive implications for treatment outcome.

\section{Data Availability}

All data generated or analyzed during this study are included in this published article.

\section{Ethical Approval}

This study was approved by the North Regional Delegation of the Ministry of Health, Garoua Regional Hospital, and surrounding health facilities (Ref. No. 01832NS/D/DRSP/N/SAG).

\section{Conflicts of Interest}

The authors declare no conflict of interest.

\section{Authors' Contributions}

RTS, CN, and EMB conceived the study and drafted the research protocol, contributed reagents and materials, and wrote the paper. PFSE, AHNK, and PBT contributed to the interpretation of the analysis and manuscript approval. MA performed tool or data analysis.

\section{Acknowledgments}

The authors thank the authorities and the health professionals of Garoua Regional Hospital and surrounding health facilities for their help in data collection and all the participants.

\section{References}

[1] J. Ferlay, M. Colombet, I. Soerjomataram et al., "Estimating the global cancer incidence and mortality in 2018: GLOBOCAN sources and methods," International journal of cancer, vol. 144, no. 8, pp. 1941-1953, 2019.

[2] T. Matsuda and A. Okuyama, "Cancer incidence rates in the world from the Cancer Incidence in Five Continents XI," Japanese journal of clinicaloncology, vol. 48, no. 2, pp. 202-203, 2018.

[3] D. R. Youlden, S. M. Cramb, C. H. Yip, and P. D. Baade, "Incidence and mortality of female breast cancer in the Asia-Pacific region," Cancer biology\&medicine, vol. 11, no. 2, pp. 101-115, 2014.

[4] J. Ferlay, I. Soerjomataram, R. Dikshit et al., "Cancer incidence and mortality worldwide: sources, methods and major patterns in GLOBOCAN 2012," International journal of cancer, vol. 136, no. 5, pp. E359-E386, 2015.

[5] J. Greenhalgh, A. Bagust, A. Boland et al., "Eribulin for the treatment of advanced or metastatic breast cancer: a NICE single technology appraisal," PharmacoEconomics, vol. 33, no. 2, pp. 137-148, 2015.

[6] F. Bray, J. Ferlay, I. Soerjomataram, R. L. Siegel, L. A. Torre, and A. Jemal, "Global cancer statistics 2018: GLOBOCAN estimates of incidence and mortality worldwide for 36 cancers in 185 countries," CA: a cancer journal for clinicians, vol. 68, no. 6, pp. 394-424, 2018.

[7] C. B. Sama, B. Dzekem, J. Kehbila et al., "Awareness of breast cancer and breast self-examination among female undergraduate students in a higher teachers training college in Cameroon," The Pan Africanmedical journal, vol. 28, 2017.

[8] B. Adedokun, Y. Zheng, P. Ndom et al., "Prevalence of Inherited Mutations in Breast Cancer Predisposition Genes among Women in Uganda and Cameroon," Cancer Epidemiology Biomarkers \& Prevention, vol. 29, no. 2, pp. 359-367, 2020.

[9] K. Azemfac, S. A. Christie, M. M. Carvalho et al., "A Community-Based Assessment of Knowledge and Practice of Breast Self- Examination and Prevalence of Breast Disease in Southwest Cameroon," Journal of cancer epidemiology, vol. 2019, Article ID 2928901, 10 pages, 2019.

[10] M. N. Okobia, C. H. Bunker, F. E. Okonofua, and U. Osime, "Knowledge, attitude and practice of Nigerian women towards 
breast cancer: a cross-sectional study," World journal of surgical oncology, vol. 4, no. 1, p. 11, 2006.

[11] O. G. E. Enow, P. Ndoh, and A. S. Doh, "Current cancer incidence and trends in Yaounde, Cameroon," Oncology, Gastroenterology and Hepatology Reports, vol. 1, 2012.

[12] S. Y. Opoku, M. Benwell, and J. Yarney, "Knowledge, attitudes, beliefs, behaviour and breast cancer screening practices in Ghana, West Africa," The Pan Africanmedical journal, vol. 11, p. 28, 2012.

[13] F. Essiben, P. Foumane, E. T. Mboudou, J. S. Dohbit, V. MveKoh, and P. Ndom, "Diagnosis and treatment of breast cancer in Cameroon: aseries of 65 cases," Le Mali medical, vol. 28 , no. $1,2013$.

[14] N. Zielonke, A. Gini, E. E. L. Jansen et al., "Evidence for reducing cancer-specific mortality due to screening for breast cancer in Europe: a systematic review," European journal of cancer, vol. 127, pp. 191-206, 2020.

[15] C. B. Haas, L. Nekhlyudov, J. M. Lee et al., "Surveillance for second breast cancer events in women with a personal history of breast cancer using breast MRI: a systematic review and meta-analysis," Breast cancer research and treatment, vol. 181, no. 2, pp. 255-268, 2020.

[16] S. Solikhah and S. Nurdjannah, "Assessment of the risk of developing breast cancer using the Gail model in Asian females: a systematic review," Heliyon, vol. 6, no. 4, p. e03794, 2020.

[17] M. Solans, D. S. M. Chan, P. Mitrou, T. Norat, and D. Romaguera, "A systematic review and meta-analysis of the 2007 WCRF/AICR score in relation to cancer-related health outcomes," Annals of oncology: official journal of the European Society for Medical Oncology, vol. 31, no. 3, pp. 352-368, 2020.

[18] K. E. Lukong, Y. Ogunbolude, and J. P. Kamdem, "Breast cancer in Africa: prevalence, treatment options, herbal medicines, and socioeconomic determinants," Breast cancer research and treatment, vol. 166, no. 2, pp. 351-365, 2017.

[19] M. A. Suh, J. Atashili, E. A. Fuh, and V. A. Eta, "Breast selfexamination and breast cancer awareness in women in developing countries: a survey of women in Buea, Cameroon," BMC research notes, vol. 5, no. 1, p. 627, 2012.

[20] F. P. Nde, J. C. Assob, T. E. Kwenti, A. L. Njunda, and T. R. Tainenbe, "Knowledge, attitude and practice of breast selfexamination among female undergraduate students in the University of Buea," BMC research notes, vol. 8, no. 1, 2015.

[21] A. F. Rositch, K. Unger-Saldana, R. J. DeBoer, A. Ng'ang'a, and B. J. Weiner, "The role of dissemination and implementation science in global breast cancer control programs: frameworks, methods, and examples," Cancer, vol. 126, Supplement 10, pp. 2394-2404, 2020.

[22] W. Insamran and S. Sangrajrang, "National Cancer Control Program of Thailand," Asian Pacific journal of cancer prevention: APJCP, vol. 21, no. 3, pp. 577-582, 2020.

[23] S. Sayed, A. K. Ngugi, M. R. Mahoney et al., "Breast cancer knowledge, perceptions and practices in a rural community in Coastal Kenya," BMC public health, vol. 19, no. 1, p. 180, 2019.

[24] R. Natarajan, D. Aljaber, D. Au et al., "Environmental exposures during puberty: window of breast cancer risk and epigenetic damage," International journal of environmentalresearch and public health, vol. 17, no. 2, 2020.
[25] A. N. Monteiro, P. Bouwman, A. N. Kousholt et al., "Variants of uncertain clinical significance in hereditary breast and ovarian cancer genes: best practices in functional analysis for clinical annotation," Journal of medicalgenetics, vol. 57, no. 8, pp. 509-518, 2020.

[26] M. E. Wood, H. T. Rehman, and I. Bedrosian, "Importance of family history and indications for genetic testing," The breast journal, vol. 26, no. 1, pp. 100-104, 2020.

[27] M. Solanki and D. Visscher, "Pathology of breast cancer in the last half century," Human Pathology, vol. 95, pp. 137-148, 2020.

[28] K. L. Britt, J. Cuzick, and K.-A. Phillips, "Key steps for effective breast cancer prevention," Nature Reviews Cancer, vol. 20, no. 8, pp. 417-436, 2020.

[29] E. Kudzawu, F. Agbokey, and C. S. K. Ahorlu, "A cross sectional study of the knowledge and practice of self-breast examination among market women at the Makola Shopping Mall, Accra, Ghana," Advances in Breast Cancer Research, vol. 5, no. 3, pp. 111-120, 2016.

[30] N. Dahiya, S. Basu, M. C. Singh, S. Garg, R. Kumar, and C. Kohli, "Knowledge and practices related to screening for breast cancer among women in Delhi, India," India, Asian Pacific journal of cancer prevention: APJCP, vol. 19, no. 1, pp. 155-159, 2018.

[31] T. O. Bello, A. I. Olugbenga-Bello, A. S. Oguntola, M. L. Adeoti, and O. M. Ojemakinde, "Knowledge and practice of breast cancer screening among female nurses and lay women in Osogbo, Nigeria," Nigeria, West African journal of medicine, vol. 30, no. 4, pp. 296-300, 2011.

[32] C. T. Nguefack, C. N'Djeudjui, J. P. N. Engbang, T. N. Nana, G. H. Ekane, and P. M. Tebeu, "Knowledge, attitude, and practice on breast cancer among health professionals in Douala references hospitals, Cameroon," Journal of cancer education: the official journal of the American Association for Cancer Education, vol. 33, no. 2, pp. 457-462, 2018.

[33] B. Lauby-Secretan, C. Scoccianti, D. Loomis et al., "Breast-cancer screening-viewpoint of the IARC Working Group," The New England journal of medicine, vol. 372, no. 24, pp. 23532358, 2015.

[34] H. Charaka, M. Khalis, S. Elfakir et al., "Organization and Evaluation of Performance Indicators of a Breast Cancer Screening Program in Meknes-Tafilalt Region," Morocco Asian Pac J Cancer Prev, vol. 17, no. 12, pp. 5153-5157, 2017.

[35] D. O. Abuidris, A. Elsheikh, M. Ali et al., "Breast-cancer screening with trained volunteers in a rural area of Sudan: a pilot study," The Lancet Oncology, vol. 14, no. 4, pp. 363370, 2013. 